\title{
Zur Torsions-Schwingungs-Rotationswechselwirkung in Rotationsspektren
}

\author{
H. DREIZLER \\ Physikalisches Institut der Universität Freiburg i. Br. \\ (Z. Naturforsch. 23 a, 1077-1085 [1968] ; eingegangen am 28. März 1968)
}

\begin{abstract}
Zur Analyse der Torsionsfeinstruktur von Rotationslinien in schwingungsangeregten Zuständen wird ein Molekülmodell diskutiert, das drei Rotations-, einen Torsions- und einen Schwingungsfreiheitsgrad besitzt. Die theoretische Formulierung ist eine Verallgemeinerung der bekannten Principal Axis Method für Molekülmodelle ohne Schwingungsfreiheitsgrad. Bei bekannter Schwingungsfrequenz tritt nur ein weiterer Potentialparameter auf.
\end{abstract}

In den letzten Jahren wurde das Hinderungspotential der $\mathrm{CH}_{3}$-Torsion einer großen $\mathrm{Zahl}$ von Molekülen aus der Torsionsfeinstruktur von Rotationslinien bestimmt. Diesen Arbeiten lag durchweg ein Modell zugrunde, bei dem das Molekül mit einem starren Rumpf und einem oder zwei starren Rotoren (top) angenähert wurde ${ }^{1}$. Der einzige in nere Freiheitsgrad bei diesem Modell ist die Torsion oder interne Rotation um eine Achse, die fest im Rumpf (frame) liegt. Das Modell besitzt außerdem noch die drei äußeren Freiheitsgrade der Rotation. Eine wesentliche Voraussetzung für die Güte dieses Modells ist die Separierbarkeit der übrigen Schwingungen von der Torsion und Rotation, die sicherlich nicht in allen Fällen gerechtfertigt ist.

Eine Aussage über die Güte des beschriebenen Modells läßt sich gewinnen, wenn man das Modell durch Hinzunahme aller Schwingungsfreiheitsgrade erweitert und nachträglich feststellt, daß das einfache Modell eine gute Näherung ist. Die gute Wiedergabe der Spektren ist nicht notwendig ein Maß für die Güte des Modells.

Das Problem eines Modells mit allen Freiheitsgraden der Torsion, Schwingung und Rotation ist theoretisch von $\mathrm{KIRTMAN}^{2}$ und Quade ${ }^{3}$ behandelt worden. Es fehlt jedoch an ausreichender experimenteller Information, um vollen Nutzen aus diesen sehr allgemeinen theoretischen Formulierungen zu ziehen.

Der Gedanke liegt deshalb nahe, das Modell bis auf die Schwingung einzuschränken, von der man die stärkste Wechselwirkung zur Torsion erwartet.

1 Zusammenfassung in H. Dreizler, Fortschr. Chem. Forsch. 10, 59 [1968].

2 B. Kirtman, J. Chem. Phys. 37, 2516 [1962].

3 C. R. Quade, J. Chem. Phys. 44, 2512 [1966].

4 K. T. Hecht u. D. M. Dennison, J. Chem. Phys. 26, 48 [1957].
Im allgemeinen wird es die Schwingung sein, die der Torsion energetisch am nächsten liegt.

In dieser Arbeit wird deshalb ein Modell behandelt, das drei Rotations-, einen Schwingungs- und einen Torsionsfreiheitsgrad besitzt. Ein analoges Modell wurde von Hecht und Dennison ${ }^{4}$ benutzt, um die Torsionsfeinstruktur des Überganges $J=0 \rightarrow 1$, $K=0 \rightarrow 0$ in drei torsionsangeregten Zuständen des Methylalkohols $\mathrm{CH}_{3} \mathrm{OH}$ zu deuten. In diesem Fall war es wegen des ungünstigen Verhältnisses $I_{\alpha} / I_{z}$ der Trägheitsmomente des Rotors $3 \mathrm{H}$ und des Rumpfes $\mathrm{COH}$ bezüglich der internen Rotationsachse ratsam, die theoretische Formulierung auf die IAM (Internal Axis Method) ${ }^{5,1}$ zu stützen. Wegen der erforderlichen molekülspezifischen NieLsen-Transformation ${ }^{6}$ wird die Methode in ihrer Anwendung schwierig.

Hier soll dagegen versucht werden, den Vorteil der allgemein breiter anwendbaren $\mathrm{PAM}^{7,5,1}$ (Principal Axis Method) weitgehend beizubehalten. Die Grenze der Anwendung wird dabei durch ein zu niedriges Hinderungspotential oder/und ein ungünstiges Verhältnis $I_{\alpha} / I_{z}$ gegeben. Sehr wertvoll für diese Arbeit war eine Mitteilung von Hirota ${ }^{8}$, auf der auch die Analyse des Rotationsspektrums in angeregten Zuständen der $\mathrm{CH}_{3}$ - und $\mathrm{C}-\mathrm{C}$-Torsion des Propionaldehyds $\mathrm{CH}_{3} \mathrm{CH}_{2} \mathrm{CHO}$ von Butcher und WILson ${ }^{9}$ beruht. Es zeigte sich allerdings, daß jedenfalls bei den Rotationsspektren des Methylthiocyanats $\mathrm{CH}_{3} \mathrm{SCN}$ in angeregten Zuständen der Biegeschwingung CSC Vereinfachungen aufgegeben und Näherungen erweitert werden mußten, die Нгвота ${ }^{8}$, Butcher und WiLson ${ }^{9}$ anwendeten.

5 C. C. Lin u. J. D. Swalen, Rev. Mod. Phys. 31, 841 [1959].

6 H. H. Nielsen, Phys. Rev. 40, 445 [1932].

7 D. R. Herschbach, J. Chem .Phys. 31, 91 [1959].

8 E. Hirota, private Mitteilung, Inhalt ist in ${ }^{9}$ skizziert.

9 S. S. Butcher u. E. B. Wilson Jr., J. Chem. Phys. 40, 1671 [1964]. 


\section{Klassische Kinetische Energie}

Im Hinblick auf die spätere Anwendung auf $\mathrm{CH}_{3} \mathrm{SCN}$ dient dieses Molekül in dieser Arbeit als Beispiel. Für die Anwendbarkeit des Modells und der Formulierungen ist aber nur notwendig:

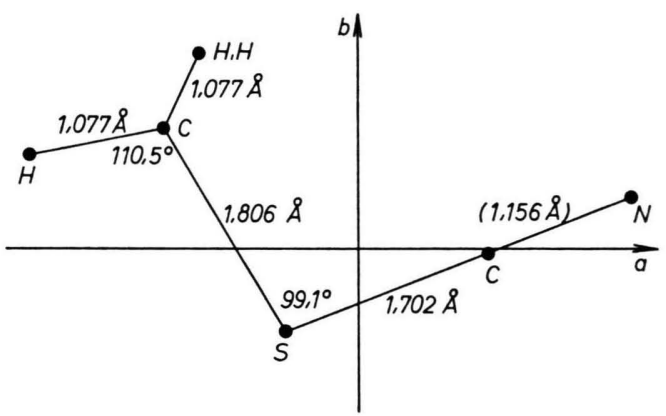

Abb. 1. Molekülmodell des $\mathrm{CH}_{3} \mathrm{SCN}$ in Hauptachsenlage mit Angabe der $r_{0}$-Struktur.

1. Der Rotor besitzt mindestens $\mathrm{C}_{3}$-Symmetrie um die interne Rotationsachse (z. B. $\mathrm{CH}_{3}$ ).

2. Bei der Schwingung werden die Atome des Rotors (also $3 \mathrm{H}$ bei $\mathrm{X}-\mathrm{CH}_{3}$ ) als starres Gebilde aus der Gleichgewichtslage entfernt. Der Rotor wird also als Ganzes gleichwertig wie die Atome des Rumpfes bei einer Schwingung behandelt.

Die Lage der Atome des Rumpfes bezüglich des Schwerpunkts C.M.M. des Moleküls sei beschrieben durch die Vektoren:

$$
\mathfrak{r}_{i}=\mathfrak{r}_{i}{ }^{0}+\mathfrak{r}_{i}{ }^{\prime} q
$$
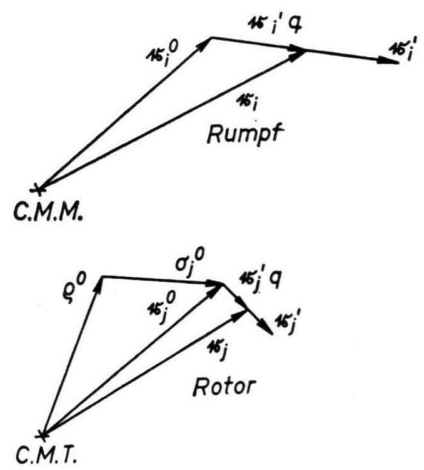

Abb. 2. Zur Definition der Ortsvektoren für Rumpf- und Rotor-Atome.

für den Rumpf und

$$
\mathfrak{r}_{j}=\mathfrak{r}_{j}{ }^{0}+\mathfrak{r}_{j}{ }^{\prime} q=\varrho^{0}+\sigma^{0}+\mathfrak{r}_{j} q
$$

für den Rotor, wobei ${ }^{0}$ die Gleichgewichtslage und $\mathrm{r}_{i}{ }^{\prime} q$ bzw. $\mathrm{r}_{j}{ }^{\prime} q$ die momentane Ausrückung aus der
Gleichgewichtslage kennzeichnet. $q$ ist eine zeitabhängige Schwingungskoordinate. $\mathfrak{r}_{i}^{\prime}$ und $\mathfrak{r}_{j}^{\prime}$ sind zeitunabhängige Vektoren, die an die Gleichgewichtslagen geheftet sind und deren Richtung und Größe von der speziellen Schwingungsform (mode) abhängt. Beim Rotor sind die Gleichgewichtslagen abhängig vom Torsionswinkel $\alpha . \varrho^{0}$ ist der Vektor vom Molekülschwerpunkt C.M.M. zum Schwerpunkt des Rotors C.M.T. $\sigma_{j}{ }^{0}$ ist der torsionswinkelabhängige Vektor vom Schwerpunkt des Rotors zu dem $j$-ten Rotoratom. Gelingt es, ein molekülbezogenes $x, y, z$-Koordinatensystem zu finden, dessen Rotation bezüglich des raumfesten $X, Y, Z$-Systems mit der Winkelgeschwindigkeit $\Omega=\left(\omega_{x}, \omega_{y}, \omega_{z}\right)$ beschrieben werden kann, so ergeben sich mit (1) und (2) die Geschwindigkeiten für die Rumpf- und Rotoratome bei separierter Translation:

$$
\begin{aligned}
& \frac{\mathrm{d} \Re_{i}}{\mathrm{~d} t}=\Omega \times \mathrm{r}_{i}+\mathrm{r}_{i}{ }^{\prime} \dot{q}, \\
& \frac{\mathrm{d} \Re_{j}}{\mathrm{~d} t}=\Omega \times \mathrm{r}_{j}+\mathfrak{\dot { \alpha }} \times \sigma_{j}+\mathrm{r}_{j}{ }^{\prime} \dot{q} .
\end{aligned}
$$

Die Vektoren $\mathfrak{r}_{i}, \mathfrak{r}_{j}$ sind in einem rotierenden Raum, dem $x, y, z$-System definiert zu denken, $\mathfrak{r}_{i}=\left(x_{i}, y_{i}\right.$, $\left.z_{i}\right)$. Im raumfesten $X, Y, Z$-System heißt der gleiche Vektor $\Re_{i}=\left(X_{i}, Y_{i}, Z_{i}\right)$. Richtung der internen Rotationsachse.

Die kinetische Energie ist:

$$
2 T=\sum_{i} m_{i}\left(\frac{\mathrm{d} \Re_{i}}{\mathrm{~d} t}\right)^{2}+\sum_{j} m_{j}\left(\frac{\mathrm{d} \Re_{j}}{\mathrm{~d} t}\right)^{2} .
$$

Durch Einsetzen von (3) und (4) in (5) erhält man:

$$
\begin{array}{rlr}
2 T=\sum_{i} m_{i}\left(\Omega \times \mathrm{r}_{i}\right)^{2} & +\sum_{j} m_{j}\left(\Omega \times \mathrm{r}_{j}\right)^{2} \\
& +\sum_{j} m_{j}\left(\mathfrak{f} \dot{\alpha} \times \sigma_{j}\right)^{2} & (6 \mathrm{a}) \\
& +\sum_{j} m_{j}\left(\Omega \times \mathrm{r}_{j}\right) \cdot\left(\mathfrak{f} \dot{\alpha} \times \sigma_{j}\right)(6 \mathrm{c}) \\
+\sum_{i} m_{i}\left(\Omega \times \mathfrak{r}_{i}\right) \cdot \mathrm{r}_{i}{ }^{\prime} \dot{q} & +\sum_{j} m_{j}\left(\Omega \times \mathfrak{r}_{j}\right) \cdot \mathrm{r}_{j}{ }^{\prime} \dot{q} & (6 \mathrm{~d}) \\
& +\sum_{j} m_{j}\left(\mathfrak{f} \dot{\alpha} \times \sigma_{j}\right) \cdot \mathrm{r}_{j}{ }_{j} \dot{q}
\end{array}
$$

Das molekülbezogene Koordinatensystem wird durch zwei Bedingungen definiert:

1. Die Schwerpunktsbedingung

$$
\sum_{i} m_{i} \mathfrak{r}_{i}+\sum_{j} m_{j} \mathfrak{r}_{j}=0
$$

ist implizit schon bei der Separation der Translation eingeführt. Der Koordinatenursprung des $x, y, z$ Systems bleibt also stets im Molekülschwerpunkt. 
2. Die Eckart-Bedingung ${ }^{10}$

$$
\sum_{i} m_{i}\left(\mathfrak{r}_{i}{ }^{0} \times \mathfrak{r}_{i}\right)+\sum_{j} m_{j}\left(\mathfrak{r}_{j}{ }^{0} \times \mathfrak{r}_{j}\right)=0
$$

oder gleichwertig

$$
\sum_{i} m_{i}\left(\mathfrak{r}_{i}{ }^{0} \times \mathrm{r}_{i}^{\prime} q\right)+\sum_{j} m_{j}\left(\mathfrak{r}_{j}{ }^{0} \times \mathrm{r}_{j}{ }^{\prime} q\right)=0
$$

orientiert ein $x, y, z$-System im Molekül. Damit verschwindet die Coriolis-Wechselwirkung (6d) in expliziter Form. Es ist nämlich mit (1) und (2)

$$
\begin{aligned}
\sum_{i} m_{i}\left(\Omega \times \mathrm{r}_{i}\right) & \cdot \mathrm{r}_{i}{ }^{\prime} \dot{q}+\sum_{j} m_{j}\left(\Omega \times \mathrm{r}_{j}\right) \cdot \mathrm{r}_{j}{ }^{\prime} \dot{q} \\
& =\left\{\sum_{i} m_{i}\left(\left(\mathrm{r}_{i}{ }^{0}+\mathrm{r}_{i}{ }^{\prime} q\right) \times \mathrm{r}_{i}{ }^{\prime} \dot{q}\right)\right. \\
& \left.+\sum_{j} m_{j}\left(\left(\mathrm{r}_{j}{ }^{0}+\mathrm{r}_{j}{ }^{\prime} q\right) \times \mathrm{r}_{j}{ }^{\prime} \dot{q}\right)\right\} \cdot \Omega .
\end{aligned}
$$

Ein Teil von $\left(6 d^{\prime}\right)$ ist Null wegen (8), der andere ist Null wegen

$$
\left(\mathrm{r}_{i}^{\prime} q \times \mathrm{r}_{i}{ }^{\prime} \dot{q}\right)=0 ;
$$

$\mathrm{r}_{i}{ }^{\prime} q$ und $\mathrm{r}_{j}{ }^{\prime} \dot{q}$ sind gleichgerichtet.

Entsprechend ist hier auch der Drehimpuls erzeugt von der Schwingung bezüglich des rotierenden Raumes $x, y, z$-System als Folge von (8) und (9) nicht vorhanden. Bei mehreren Schwingungsfreiheitsgraden ist dieser Drehimpuls durch (8) nur minimalisiert.

Da nach Konstruktion des Modells der Rotor sich als Ganzes bei einer Schwingung bewegt, lassen sich die Verrückungsvektoren bei jeglicher nicht zu großen Schwingungsauslenkung zerlegen in

$$
\mathfrak{r}_{j}^{\prime}=\varrho^{\prime}+\sigma_{j}^{\prime}=\varrho^{\prime}+\delta \beta \times \sigma_{j}{ }^{0} .
$$

$\varrho^{\prime}$ ist der Translationsanteil, der gleich der Translation des Rotorschwerpunktes ist. $\delta \beta \times \sigma_{j}{ }^{0}$ ist der Rotationsanteil, der von den $\mathfrak{r}_{j}^{\prime}$ beschriebenen Bewegung. $\delta \beta$ ist ein Drehwinkel um eine Achse durch den Rotorschwerpunkt.

$\mathrm{Da}$

$$
\sum_{j} m_{j} \sigma_{j}^{0}=0
$$

und

$$
\sum_{j} m_{j}\left(\sigma_{j}^{0}+\sigma_{j}{ }^{\prime}\right)=0
$$

folgt aus (8) mit (10)

$$
\begin{aligned}
\sum_{i} m_{i}\left(\mathrm{r}_{i}{ }^{0} \times \mathrm{r}_{i}{ }^{\prime} q\right) & +\left(\varrho^{0} \times \varrho^{\prime}\right) \sum_{j} m_{j} \\
& +\sum_{j} m_{j}\left(\sigma_{j}{ }^{0} \times\left(\delta \beta \times \sigma_{j}{ }^{0}\right)\right)=0 .
\end{aligned}
$$

In (13) sind allein die $\sigma_{j}{ }^{0}$ in ihrer Orientierung vom Torsionswinkel $\alpha$ abhängig, ihr Betrag ist konstant.

\footnotetext{
10 C. Eckart, Phys. Rev. 47, 552 [1935].
}

Wichtig aber ist, daß die Bedingung (13) unabhängig von $\alpha$ ist. Zum Beweis genügt es, die letzte Summe in (13) zu betrachten. Mit $\delta \beta=\delta \beta^{\prime}+\delta \beta^{\prime \prime}$, einer Zerlegung der Drehung $\delta \beta$ in eine mit einer Achse senkrecht und parallel zu der von den $\sigma_{j}{ }^{0}$ aufgespannten Ebene, erhält man:

$$
\begin{aligned}
\sum_{j} m_{j}\left(\sigma_{j}{ }^{0} \times\left(\delta \beta \times \sigma_{j}{ }^{0}\right)\right) & \\
=\sum_{j} m_{j}\left(\left(\sigma_{j}{ }^{0} \cdot \sigma_{j}{ }^{0}\right) \delta \beta\right. & -\left(\delta \beta^{\prime} \cdot{\sigma_{j}}^{0}\right){\sigma_{j}{ }^{0}}^{0} \\
& \left.-\left(\delta \beta^{\prime \prime} \cdot{\sigma_{j}}^{0}\right){\sigma_{j}}^{0}\right),
\end{aligned}
$$

wobei die mittlere Summe gleich Null ist. Die erste Summe ist von $\alpha$ unabhängig, die letzte ist es nur, wenn $m_{j}=m$ und der Rotor mindestens $\mathrm{C}_{3}$-Symmetrie hat. Es ist dann für $\mathrm{C}_{3}$-Symmetrie und $\left|\sigma_{j}{ }^{0}\right|=\sigma^{0}$

$$
\sum_{j} m_{j}\left(\delta \beta^{\prime \prime} \cdot \sigma_{j}{ }^{0}\right) \sigma_{j}^{0}=\frac{3}{2} m\left(\sigma^{0}\right)^{2} \delta \beta^{\prime \prime}
$$

Da also (13) unabhängig von $\alpha$ ist, sind die Verrückungen $\mathfrak{r}_{j}^{\prime}$ und $\varrho^{\prime}$ ebenfalls von $\alpha$ unabhängig.

Ein geeignetes EcKart-System ist ein System, das bei $q=0$, d. h. bei Gleichgewichtslage der Atome, Hauptachsensystem ist.

Für die kinetische Energie erhält man dann in tensorieller Form

$$
\begin{gathered}
T=\frac{1}{2} \Omega_{t s}^{+} \cdot I \cdot \Omega_{t s} \\
\frac{1}{2} I=\left(\begin{array}{ccccc}
I_{x x} & I_{x y} & I_{x z} & \lambda_{x} I_{\alpha} & 0 \\
I_{y x} & I_{y y} & I_{y z} & \lambda_{y} I_{a} & 0 \\
I_{z x} & I_{z y} & I_{z z} & \lambda_{z} I_{\alpha} & 0 \\
\lambda_{x} I_{\alpha} & \lambda_{y} I_{1} & \lambda_{z} I_{\alpha} & I_{\alpha} & S \\
0 & 0 & 0 & S & G^{-1}
\end{array}\right) .
\end{gathered}
$$

In (17) ist:

$\Omega_{t s}^{\dagger}=\left(\omega_{x}, \omega_{y}, \omega_{z}, \dot{\alpha}, \dot{q}\right)$,

$I_{g g}=\sum_{k} m_{k}\left(g_{k}^{\prime 2}+g_{k}^{\prime \prime 2}\right), \quad g, g^{\prime}, g^{\prime \prime}=x, y, z$ zyklisch

$I_{g g^{\prime}}=-\sum_{k} m_{k} g g^{\prime}$,

$I_{\alpha}$ Trägheitsmoment des Rotors um die interne Rotationsachse,

$\lambda_{g} \quad$ Richtungskosinus zwischen interner Rotationsachse und $g$-Achse $(g=x, y, z)$ des EckartSystems

$S=\mathfrak{l} \cdot \sum_{j} m_{j}\left(\sigma_{j}^{0} \times\left(\delta \beta \times \sigma_{j}^{0}\right)\right)$

$G^{-1}=\sum_{i} m_{i} \mathrm{r}_{i}^{\prime 2}+3 m\left(\varrho^{\prime 2}+\left(\sigma^{0}\right)^{2}\left(\delta \beta^{\prime 2}+\frac{1}{2} \delta \beta^{\prime \prime 2}\right)\right)$ mit (18e)

$\delta \beta=\delta \beta^{\prime}+\delta \beta^{\prime \prime},\left|\sigma_{j}{ }^{0}\right|=\sigma^{0} \quad$ und $\mathrm{C}_{3}$-Symmetrie. 
Betrachtet man eine Schwingung, bei der $\delta \beta=\delta \beta^{\prime \prime}$, so wird $S=0$, da $\perp \delta \beta^{\prime \prime}$. Die Bedingung $\delta \beta=\delta \beta^{\prime \prime}$ hat zur Folge, daß keine Drehimpulskomponente in Richtung der internen Rotationsachse erzeugt von der Schwingung existiert. Es ist nämlich mit (14) und (15)

$$
\mathfrak{f} \cdot \sum_{j} m_{j}\left(\sigma_{j} \times \sigma_{j}^{\prime} \dot{q}\right)=0 .
$$

Es ist zu beachten, daß alle Größen im Tensor (17) unabhängig von $\alpha$ sind, was eine direkte Folge der $\mathrm{C}_{3}$-Symmetrie des Rotors ist. Es sind aber alle Größen bis auf $I_{a}, S$ und $G^{-1}$ Funktionen von $q$. Sie lassen sich in der Form

$$
\begin{aligned}
& I_{g g}=I_{g g}^{0}+I_{g g}^{\prime} q+I_{g g}^{\prime \prime} q^{2}, \\
& I_{g g^{\prime}}=\quad I_{g g^{\prime}}^{\prime} q+I_{g g^{\prime}}^{\prime \prime} q^{2}, \\
& \lambda_{g}=\lambda_{g}^{0}+\lambda_{g}^{\prime} q
\end{aligned}
$$

entwickeln. $I_{g g^{\prime}}^{0}=0$ wegen der obigen Wahl des EcKarT-Systems. Es ist Hauptträgheitsachsensystem in der Gleichgewichtslage.

Hat das Molekül eine Symmetrieebene, etwa die Ebene $y z$, so ist $\lambda_{x}{ }^{0}=0$.

Ausgedrückt mit den Impulsen

$$
\mathfrak{P}_{t s}^{\dagger}=\left(P_{x}, P_{y}, P_{z}, p_{a}, p_{q}\right)
$$

mit

$$
P_{g}=\partial T / \partial \omega_{g} ; \quad p_{\alpha}=\partial T / \partial \dot{\alpha} ; \quad p_{q}=\partial T / \partial \dot{q}(22 \mathrm{~b}-\mathrm{d})
$$

erhält man für die kinetische Energie

$$
T=\frac{1}{2} \mathfrak{F}_{t s}^{\dagger} \cdot I^{-1} \cdot \mathfrak{P}_{t s}
$$

mit

$$
\begin{aligned}
& \frac{1}{2} I^{-1}=\left(\begin{array}{ccccc}
C & D_{x y} & D_{x z} & -Q_{x} & R_{x} \\
D_{y x} & B & D_{y z} & -Q_{y} & R_{y} \\
D_{z x} & D_{z y} & A & -Q_{z} & R_{z} \\
-Q_{x} & -Q_{y} & -Q_{z} & F & -L \\
R_{x} & R_{y} & R_{z} & -L & M / 2
\end{array}\right), \\
& C \quad=\frac{1}{2}\left(a_{x x}+b_{x x}\right), \\
& B \quad= \frac{1}{2}\left(a_{y y}+b_{y y}\right), \\
& A \quad=\frac{1}{2}\left(a_{z z}+b_{z z}\right), \\
& D_{g g^{\prime}}=\frac{1}{2}\left(a_{g g^{\prime}}+b_{g g^{\prime}}\right), g, g^{\prime}=x, y, z, g \neq g^{\prime}, \\
& Q_{g}=\left(1 / 2 r_{0}\right) \sum_{g^{\prime}} \lambda_{g^{\prime}} a_{g g^{\prime}}, \\
& R_{g}=\left[S /\left(2 r_{0} G^{-1}\right)\right] \sum_{g^{\prime}} \lambda_{g^{\prime}} a_{g g^{\prime}}, \\
& F \quad=1 /\left(2 I_{a} r_{0}\right), \\
& L \quad=S /\left(2 I_{a} r_{0} G^{-1}\right), \\
& M \quad=r /\left(r_{0} G^{-1}\right),
\end{aligned}
$$

$$
\begin{aligned}
r_{0} \quad= & r-S^{2} /\left(I_{a} G^{-1}\right) \\
= & 1-I_{a} \sum_{g g^{\prime}} \lambda_{g} \lambda_{g^{\prime}} a_{g g^{\prime}}-S^{2} /\left(I_{a} G^{-1}\right), \\
\left\|a_{g g^{\prime}}\right\| & =\left\|I_{g g^{\prime}}\right\|-1, \\
\left\|b_{g g^{\prime}}\right\| & =\left(I_{a} / r_{0}\right)\left\|\sum_{g^{\prime \prime} g^{\prime \prime \prime}} \lambda_{g^{\prime \prime}} \lambda_{g^{\prime \prime \prime}} a_{g g^{\prime \prime}} a_{g^{\prime \prime \prime} g^{\prime}}\right\| .
\end{aligned}
$$

Es sind jetzt alle Größen des Tensors (24) Funktionen von $q$, aber unabhängig von $\alpha$.

Der Tensor (24) wird wesentlich einfacher, wenn man sich auf Moleküle mit einer Symmetrieebene (hier $y z$-Ebene) und eine Schwingung in dieser Ebene beschränkt. Im Tensor (17) ist dann:

$$
I_{x y}=0 ; \quad I_{x z}=0 ; \quad \lambda_{x}=0 ; \quad S=0 .
$$

Damit vereinfacht sich der Tensor (24) zu:

$$
\frac{1}{2} I^{-1}=\left(\begin{array}{ccccc}
C & 0 & 0 & 0 & 0 \\
0 & B & D_{y z}-Q_{y} & 0 \\
0 & D_{z y} & -Q_{z} & -Q_{z} & 0 \\
0 & -Q_{y} & A & F & 0 \\
0 & 0 & 0 & 0 & M / 2
\end{array}\right)
$$

mit:

$A=I_{y y} /(2 \Delta)+2 I_{a} r Q_{z}{ }^{2}$,

$B=I_{z z} /(2 \Delta)+2 I_{a} r Q_{y}^{2}$,

$C=1 /\left(2 I_{x x}\right)$,

$D_{y z}=-I_{y z} /(2 \Delta)+2 I_{\alpha} r Q_{y} Q_{z}$,

$Q_{y}=\left(\lambda_{y} I_{z z}-\lambda_{z} I_{z y}\right) /(2 r \Delta)$,

$Q_{z}=\left(\lambda_{z} I_{y y}-\lambda_{y} I_{y z}\right) /(2 r \Delta)$,

$F=1 /\left(2 I_{a} r\right)$,

$M=1 / G^{-1}$,

$r=1-I_{a}\left(\lambda_{z}^{2} I_{y y}-2 \lambda_{y} \lambda_{z} I_{y z}+\lambda_{y}^{2} I_{z z}\right) / \Delta$,

$\Delta=I_{y y} I_{z z}-I_{z y} I_{y z}$.

Bis auf $M$ sind alle Größen des Tensors (26) Funktionen von $q$. Später werden sie nach $q$ entwickelt.

Bei der Aufstellung des Hamilton-Operators wird auf die Struktur von (26) zurückgegriffen werden.

\section{Potentielle Energie}

Für die potentielle Energie verwendet man einen Ansatz. Er wird von der Bedingung, daß bei $q=0$ und $\alpha=\frac{2}{3} \pi n$ Minima sein sollen und daß eine konstante, von $\alpha$ und $q$ unabhängige potentielle Energie unwesentlich ist, modifiziert. Der Ansatz ist

$$
\begin{aligned}
V(\alpha, q)= & k_{0}+k_{1} q+k_{2} q^{2}+\ldots \\
& -\left(c_{0}+c_{1} q+c_{2} q^{2}+\ldots\right) \cos 3 \alpha \\
& +\left(s_{0}+s_{1} q+s_{2} q^{2}+\ldots\right) \sin 3 \alpha \\
& +\ldots .
\end{aligned}
$$


Höhere Glieder werden vernachlässigt. Mit den beiden Bedingungen erhält man:

$$
\begin{aligned}
V(\alpha, q)= & c_{0}(1-\cos 3 \alpha)+c_{1}(1-\cos 3 \alpha) q \\
& +s_{1}(\sin 3 \alpha) q+k_{2} q^{2} .
\end{aligned}
$$

Besitzt das Molekül eine Symmetrieebene und ist die Schwingung totalsymmetrisch, so vereinfacht sich $V(\alpha, q)$ weiter:

$V(\alpha, q)=c_{0}(1-\cos 3 \alpha)+c_{1}(1-\cos 3 \alpha) q+k_{2} q^{2}$

oder :

$$
\begin{aligned}
V(\alpha, q)= & \frac{1}{2} V_{3}(1-\cos 3 \alpha)+\frac{1}{2}\left(\omega^{2} / M\right) q^{2} \\
& +V_{\mathrm{c}}^{\prime} q(1-\cos 3 \alpha) .
\end{aligned}
$$

Der erste Teil ist das Hinderungspotential der Höhe $V_{3}$, der zweite ist das Potential der harmonischen Schwingung mit der Kreisfrequenz $\omega$ und der inversen reduzierten „Masse“ $M$ und der dritte Teil beschreibt eine Wechselwirkung zwischen Torsion und Schwingung. Bei Beschränkung von $\alpha$ auf kleine Amplituden erweist sich $V_{\mathrm{c}}{ }^{\prime}$ als eine kubische Kraftkonstante.

\section{Hamilton-Operator}

Bei der Aufstellung des Hamilton-Operators ist nach Podolsky ${ }^{11}$ zu beachten, daß der Tensor (24) und (26) keine Konstanten, sondern Funktionen von $q$ enthält. Der Torsionswinkel $\alpha$ ist infolge der $\mathrm{C}_{3}$-Symmetrie des Rotors nicht enthalten.

Im folgenden wird nur der einfache Fall, ein Molekül mit Symmetrieebene und totalsymmetrischer Schwingung behandelt. Für andere Fälle verläuft die Ableitung analog aber komplizierter. In der allgemeinen Form des Hamilton-Operators

$$
H=\frac{1}{2} g^{-1 / 4} \sum_{i, k} P_{i} g^{1 / 2} g^{i k} P_{k} g^{-1 / 4}+V\left(q_{k}\right)
$$

für unseren Fall spezialisiert mit

$g=\operatorname{det} I$,

$\left\|g^{i k}\right\|=I^{-1}$,

$$
\begin{aligned}
& P_{i}=P_{x}, P_{y}, P_{z}, p_{a}, p_{q}, \\
& p_{a}=\frac{\hbar}{i} \frac{\partial}{\partial \alpha}, p_{q}=\frac{\hbar}{i} \frac{\partial}{\partial q},
\end{aligned}
$$

$P_{g}$ Komponenten des Gesamtdrehimpulses, kommutieren alle Operatoren mit den Koeffizienten $g^{i k}$ und $g$ außer dem Operator $p_{q}$.

Da aber in dem Spezialfall (26) nur ein Element der fünften Spalte und Zeile ungleich Null ist, bedarf nur ein Summand in (31) einer genaueren Betrachtung. $\mathrm{Da} \beta$ in der klassischen Energie $M$ unabhängig von $q$ ist, hebt diese Tatsache nicht auf.

Mit

$$
C_{55}=g / G^{-1}
$$

erhält man für

$$
\begin{aligned}
& \frac{1}{2} g^{-1 / 4} p_{q} g^{1 / 2} g^{55} p_{q} g^{-1 / 4} \\
& \quad=\frac{1}{2} M\left(p_{q}^{2}-\frac{1}{4} C_{55}^{-1}\left\{p_{q}{ }^{2} C_{55}\right\}+\frac{3}{16} C_{55}^{-2}\left\{p_{q} C_{55}\right\}^{2}\right) .
\end{aligned}
$$

Die geschweiften Klammern deuten an, daß der Operator nur auf den Klammerinhalt wirken soll. Den ersten Summanden erhielte man allein, wenn das Verfahren nach Podolsky nicht beachtet würde. Die beiden letzten Summanden sind Funktionen von $q$, da $C_{55}$ eine Funktion von $q$ ist. Sie werden in $W^{0}+W^{\prime} q+W^{\prime \prime} q^{2}$ entwickelt. Die Konstante $W^{\mathbf{0}}$ wird weggelassen werden. Der Gesamtoperator hat jetzt mit nach $q$ entwickelten Koeffizienten folgende Form:

$$
\begin{aligned}
& H=\left(\underline{C^{0}}+C^{\prime} q+C^{\prime \prime} q^{2}\right) \underline{P_{x}^{2}} \\
& +\left(\underline{B}^{0}+B^{\prime} q+B^{\prime \prime} q^{2}\right) \underline{P_{y}}{ }^{2} \\
& +\left(\underline{A^{0}}+A^{\prime} q+A^{\prime \prime} q^{2}\right) \underline{P_{z}^{2}} \\
& +\left(D_{y z}^{\prime} q+D^{\prime \prime}{ }_{y z} q^{2}\right)\left(P_{y} P_{z}+P_{z} P_{y}\right) \\
& +\left(\underline{F^{0}}+F^{\prime} q+F^{\prime \prime} q^{2}\right) \underline{p_{\alpha}^{2}}+\underline{\frac{1}{2} V_{3}(1-\cos 3 \alpha)} \\
& -2\left(Q_{y}{ }^{0}+Q_{y}^{\prime} q+Q^{\prime \prime}{ }_{y} q^{2}\right) p_{\alpha} P_{y} \\
& -2\left(Q_{z}{ }^{0}+Q^{\prime}{ }_{z} q+Q^{\prime \prime}{ }_{z} q^{2}\right) p_{\alpha} P_{z} \\
& +\underline{\frac{1}{2} M p_{q}^{2}+\frac{1}{2}\left(\omega^{2} / M\right) q^{2}} \\
& +V_{\mathrm{c}}{ }^{\prime} q(1-\cos 3 \alpha) \\
& +W^{\prime} q+W^{\prime \prime} q^{2}
\end{aligned}
$$

wobei die in Klammern gesetzten Polynome in $q$ mit den Größen aus (27) in der folgenden Weise verknüpft sind:

$$
X^{0}+X^{\prime} q+X^{\prime \prime} q^{2}=X
$$

mit $X=A, B, C, D_{y z}, F, Q_{y}, Q_{z}$. Aus (36) kann man Näherungsoperatoren der reinen Rotation, Torsion und Schwingung isolieren, was durch Unter-

11 B. Podolsky, Phys. Rev. 32, 812 [1928] ; siehe auch E. B. Wilson JR., I. C. Decius u. P. C. Crass, Molecular Vibrations, McGraw-Hill Book Co. Inc., New York 1955, Kap. 11-3. 
streichen gekennzeichnet ist. Die reine TorsionsRotations-Wechselwirkung des Modells ohne Schwingungsfreiheitsgrade ist in (36) ebenfalls enthalten. Wesentlich neu sind die Wechselwirkungsoperatoren zwischen Rotation, Torsion und Schwingung.

\section{Behandlung des Hamilton-Operators}

Mit den Eigenwerten des Operators (36) läßt sich ein Termschema aufbauen, mit dem die gemessenen Spektren verglichen werden können.

Es ist möglich, eine Energiematrix von (36) in einem Basissystem $R(\varphi, \vartheta, \chi) U(\alpha) H(q)$ aus den Eigenfunktionen der Näherungsoperatoren

$$
\begin{aligned}
& C^{0} P_{x}^{2}+B^{0} P_{y}^{2}+A^{0} P_{z}^{2}, \\
& F^{0} p_{a}{ }^{2}+\frac{1}{2} V_{3}(1-\cos 3 \alpha), \\
& \frac{1}{2} M{p_{q}}^{2}+\frac{1}{2}\left(\omega^{2} / M\right) q^{2}
\end{aligned}
$$

aufzustellen. Die Matrix hätte aber für höhere Werte der Drehimpulsquantenzahl $J$ einen solchen Rang, daß die numerische Diagonalisierung mit den bestehenden Rechenanlagen zu einem kaum vertretbaren Aufwand führt.

Eine andere Möglichkeit ist, den Teil von (36), der keine Rotationsoperatoren enthält, also (36e), $(36 \mathrm{~h}-\mathrm{j})$ im Basissystem $U(\alpha) H(q)$ aus Eigenfunktionen der Näherungsoperatoren (39) und (40) zu bearbeiten. Eine Diagonalisierung der Matrix von $(36 \mathrm{e}),(36 \mathrm{~h}-\mathrm{j})$ führt dann zu einem neuen Basissystem $R(\varphi, \vartheta, \chi) F(\alpha, q)$ für den Gesamtoperator (36). In dem Basissystem $R(\varphi, \vartheta, \chi) F(\alpha, q)$ sind nur die mit $P_{g}$ behafteten Wechselwirkungsoperatoren aus $(36 \mathrm{a}-\mathrm{d})$, (36f) und $(36 \mathrm{~g})$ nicht diagonal. Eine genäherte Diagonalisierung läßt sich analog zur PAM ${ }^{7,5}$ mit einer van Vleck-Transformation ${ }^{12-15}$ und nachfolgender $K$-Diagonalisierung erreichen. Diese Verfahrensweise wird zur Zeit bearbeitet. Die Untersuchung ist aber noch nicht abgeschlossen.

Die letzte Möglichkeit ist, die Matrix von (36) im Basissystem $R(\varphi, \vartheta, \chi) U(\alpha) H(q)$ der Näherungsoperatoren (38), (39) und (40) aufzustellen

12 E. C. Kemble, Fundamental Principles of Quantum Mechanics, Dover Publications Inc., New York 1958, p. 394.

13 J. H. van Vleck, Phys. Rev. 33, 487 [1929].

14 O. M. Jordahl, Phys. Rev. 45, 87 [1934].

15 D. R. Herschbach, Tables on the Internal Rotation Problem, Dept. of Chemistry, Harvard University 1957. und Methoden der Störungsrechnung anzuwenden. Dieser Weg soll näher beschrieben werden.

Da die Eigenfunktionen des Hamilton-Operators (38) eines asymmetrischen Kreisels nicht explizit zur Verfügung stehen, ist es praktisch, mit den Eigenfunktionen eines symmetrischen Kreisels zu arbeiten. (38) wird also ersetzt durch:

$$
H_{R s}^{0}=\frac{1}{2}\left(C^{0}+B^{0}\right)\left(P_{x}^{2}+P_{y}^{2}\right)+A^{0} P_{z}^{2}
$$

mit den Eigenfunktionen $\psi_{J K M}^{\times}(\varphi, \vartheta, \chi)$. Damit spaltet der zusätzliche Störoperator

$$
H_{R a}^{0}=\frac{1}{2}\left(C^{0}-B^{0}\right)\left(P_{x}^{2}-P_{y}^{2}\right)
$$

ab.

Im Basissystem $\psi_{J K M}^{\times}(\varphi, \vartheta, \chi) U_{v_{a} \sigma}(\alpha) H_{v_{q}}(q)$ ergibt (36) eine in $J, M, \sigma$ diagonale und in $K, v_{\alpha}, v_{q}$ nicht diagonale Matrix. Wenn die Rotationsenergiedifferenzen klein gegen die Torsions-Schwingungsdifferenzen sind, kann man wie bei PAM mit Hilfe einer van Vleck-Transformation ${ }^{12-16}$ zunächst eine genäherte $v_{a} v_{q}$-Diagonalisierung für ein $v_{a} v_{\mathrm{q}}$ - $\mathrm{Ni}$ veau durchführen. Sind die Rotationsenergiedifferenzen in den Nennern der Störsummen vernachlässigbar, so gelangt man auf diese Weise zu einem effektiven Rotationsoperator.

Lassen sich die Rotationsenergiedifferenzen nicht vernachlässigen, so kann man mit Hilfe der Nennerkorektur nach Herschbach ${ }^{7,17}$ eine Korrektur anbringen. Über die für diesen komplizierten Fall notwendige Verallgemeinerung soll gesondert berichtet werden. Auch in diesem Fall gelangt man zu einem effektiven Rotationsoperator.

Für einen Operator

$$
H^{0}+\lambda H^{\prime}
$$

mit den Eigenwerten $E$ des Operators $H^{0}$ sind die verwendeten Formeln der van Vleck-Transformation bis zur vierten Ordnung mit der Kurzschreibweise ${ }^{18}$

$$
\begin{aligned}
\left(v_{a} v_{q} K\left|H^{\prime}\right| v_{a} v_{q} K^{\prime}\right) & =H_{a a^{\prime}}^{\prime}, \\
\left(v_{a} v_{q} K\left|H^{\prime}\right| v_{a}^{\prime} v_{q}^{\prime} K^{\prime}\right) & =H_{a b}^{\prime}
\end{aligned}
$$

1. Ordnung:

$$
\lambda H_{a a^{\prime}} ;
$$

16 M. Hayashi, A Review on the Energy Levels for Internal Torsion and Over-all Rotation of Two Top Molecules, University of Hiroshima 1964, App. I, Private Mitteilung.

17 D. Stelman, J. Chem. Phys. 41, 2111 [1964].

18 Die Quantenzahlen $\sigma J M$ werden unterdrückt, da die Matrix in diesen Quantenzahlen diagonal ist. 
2. Ordnung:

3. Ordnung:

$$
\left(\lambda^{2} / 2\right)\left\{\sum_{b} H_{a b}^{\prime} H_{b a^{\prime}}^{\prime} /\left(E_{a}-E_{b}\right)+\sum_{b} H^{\prime}{ }_{a b} H^{\prime}{ }_{b a^{\prime}} /\left(E_{a^{\prime}}-E_{b}\right)\right\}
$$

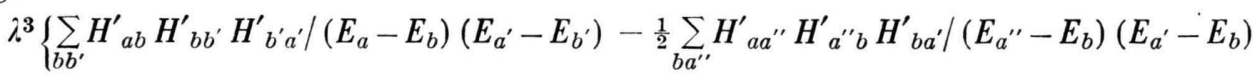

$$
\begin{aligned}
& \left.-\frac{1}{2} \sum_{b a^{\prime \prime}} H^{\prime}{ }_{a b} H_{b a^{\prime \prime}}^{\prime} H_{a^{\prime \prime} a^{\prime}}^{\prime} /\left(E_{a}-E_{b}\right)\left(E_{a^{\prime \prime}}-E_{b}\right)\right\} \text {; }
\end{aligned}
$$

4. Ordnung:

$$
\begin{gathered}
\left(-\lambda^{4} / 8\right)\left\{\sum_{a^{\prime \prime} b b^{\prime}} H_{a b}^{\prime} H_{b a^{\prime \prime}}^{\prime} H_{a^{\prime \prime} b^{\prime}}^{\prime} H_{b^{\prime} a^{\prime}}^{\prime}\right\}\left\{3 /\left(E_{a}-E_{b}\right)\left(E_{a^{\prime \prime}}-E_{b^{\prime}}\right)\left(E_{a^{\prime}}-E_{b^{\prime}}\right)+3 /\left(E_{a}-E_{b}\right)\left(E_{a^{\prime}}-E_{b^{\prime}}\right)\left(E_{a^{\prime \prime}}-E_{b}\right)\right. \\
\left.+1 /\left(E_{a^{\prime}}-E_{b^{\prime}}\right)\left(E_{a^{\prime \prime}}-E_{b}\right)\left(E_{a^{\prime \prime}}-E_{b^{\prime}}\right)+1 /\left(E_{a}-E_{b}\right)\left(E_{a^{\prime \prime}}-E_{b}\right)\left(E_{a^{\prime \prime}}-E_{b^{\prime}}\right)\right\} \\
+\left(\lambda^{4} / 2\right)\left\{\sum_{b} 1 /\left(E_{a}-E_{b}\right)+1 /\left(E_{a^{\prime}}-E_{b}\right)\right\}\left\{\sum_{a^{\prime \prime}} H^{\prime}{ }_{a a^{\prime \prime}} H_{a^{\prime \prime} b}^{\prime} /\left(E_{b}-E_{a^{\prime \prime}}\right)+\sum_{b^{\prime}}^{\prime} H_{a b^{\prime}}^{\prime} H_{b^{\prime} b}^{\prime} /\left(E_{a}-E_{b^{\prime}}\right)\right\} \\
\left\{\sum_{b^{\prime \prime}} H_{b b^{\prime \prime}}^{\prime} H_{b^{\prime \prime} a^{\prime}}^{\prime} /\left(E_{a^{\prime}}-E_{b^{\prime \prime}}\right)-\sum_{a^{\prime \prime \prime}} H_{b a^{\prime \prime}}^{\prime} H_{a^{\prime \prime \prime} a^{\prime}}^{\prime} /\left(E_{a^{\prime \prime \prime}}-E_{b}\right)\right\}
\end{gathered}
$$

(45) bis (48) geben also die Beiträge für das $\left(a \mid a^{\prime}\right)$-Element. In unserem Fall ist $H^{0}$ mit den Näherungsoperatoren (39), (40) und (41), $\lambda H^{\prime}$ mit den restlichen Operatoren aus (36) zu identifizieren, formal also:

$$
\lambda H^{\prime}=\lambda \sum_{i}^{n} H_{i}^{\prime}
$$

Werden die typischen Nennerdifferenzen angenähert durch:

$$
\left(E_{a}-E_{b}\right) \equiv\left(E_{v^{\alpha} \sigma}-E_{v^{\prime} \alpha^{\sigma}}\right)+\left(E_{v_{q}}-E_{v^{\prime} q}\right)+\left(E_{J K}-E_{J^{\prime} K^{\prime}}\right) \approx\left(E_{v_{\alpha} \sigma}-E_{v^{\prime} \alpha^{\sigma}}\right)+\left(E_{v_{q}}-E_{v^{\prime} q}\right)
$$

so läßt sich der Rotationsanteil in den Störsummen abseparieren. Der Torsions-Schwingungsanteil ist dann für jedes Element der $v_{\alpha} v_{q}$-Submatrix gleich. Welches Element dieser $v_{\alpha} v_{q}$-Submatrix einen Beitrag erhält, zeigen die Quantenzahlen des abseparierten Rotationsteils. (45) bis (48) wird mit separierten Torsions-Schwingungs- und Rotationsanteilen $T V$ und $R$ für eine Operatorkombination $H_{i}{ }^{\prime 19}$ :

für eine Operatorkombination $H_{i}^{\prime} H_{j}^{\prime}$ :

$$
\lambda\left(T_{i} V_{i}\right)_{a a} \cdot\left(R_{i}\right)_{a a^{\prime}} \equiv C_{i} h_{i}\left(r_{i}\right)_{K K^{\prime}},
$$

$$
\lambda^{2}\left(\sum_{b}\left(T_{i} V_{i}\right)_{a b}\left(T_{j} V_{j}\right)_{b a} /\left(E_{a}-E_{b}\right)\right) \cdot\left(R_{i} R_{j}\right)_{a a^{\prime}} \equiv \frac{1}{\frac{9}{4} F^{0}} C_{i j}\left(h_{i} h_{j} / \Delta\right) \cdot\left(r_{i} r_{j}\right)_{K K^{\prime}},
$$

für eine Operatorkombination $H_{i}{ }^{\prime} H_{j}{ }^{\prime} H_{k}{ }^{\prime}$ :

$$
\begin{gathered}
\lambda^{3}\left\{\sum_{b b^{\prime}}\left(T_{i} V_{i}\right)_{a b}\left(T_{j} V_{j}\right)_{b b^{\prime}}\left(T_{k} V_{k}\right)_{b^{\prime} a} /\left(E_{a}-E_{b}\right)\left(E_{a}-E_{b^{\prime}}\right)-\frac{1}{2}\left(T_{i} V_{i}\right)_{a a} \sum_{b}\left(T_{j} V_{j}\right)_{a b}\left(T_{k} V_{k}\right)_{b a} /\left(E_{a}-E_{b}\right)^{2}\right. \\
\left.-\frac{1}{2}\left(\sum_{b}\left(T_{i} V_{i}\right)_{a b}\left(T_{j} V_{j}\right)_{b a} /\left(E_{a}-E_{b}\right)^{2}\right)\left(T_{k} V_{k}\right)_{a a}\right\} \cdot\left(R_{i} R_{j} R_{k}\right)_{K K^{\prime}} \\
\equiv \frac{1}{\left(\frac{9}{4} F^{0}\right)^{2}} C_{i j k}\left\{h_{i} h_{j} h_{k} / \Delta \Delta^{\prime}-\frac{1}{2} h_{i}\left(h_{j} h_{k} / \Delta^{2}\right)-\frac{1}{2}\left(h_{i} h_{j} / \Delta^{2}\right) h_{k}\right\} \cdot\left(r_{i} r_{j} r_{k}\right)_{K K^{\prime}},
\end{gathered}
$$

für eine Operatorkombination $H_{i}{ }^{\prime} H_{j}{ }^{\prime} H_{k}{ }^{\prime} H_{l}$ :

$$
\begin{aligned}
& \lambda^{4}\left\{-\frac{1}{2}\left(\sum_{b}\left(T_{i} V_{i}\right)_{a b}\left(T_{j} V_{j}\right)_{b a} /\left(E_{a}-E_{b}\right)\right)\left(\sum_{b^{\prime}}\left(T_{k} V_{k}\right)_{a b^{\prime}}\left(T_{l} V_{l}\right)_{b^{\prime} a} /\left(E_{a}-E_{b^{\prime}}\right)^{2}\right)\right. \\
& -\frac{1}{2}\left(\sum_{b}\left(T_{i} V_{i}\right)_{a b}\left(T_{j} V_{j}\right)_{b a} /\left(E_{a}-E_{b}\right)^{2}\right)\left(\sum_{b^{\prime}}\left(T_{k} V_{k}\right)_{a b^{\prime}}\left(T_{l} V_{l}\right)_{b^{\prime} a} /\left(E_{a}-E_{b^{\prime}}\right)\right) \\
& -\sum_{b b^{\prime \prime}}\left(T_{i} V_{i}\right)_{a a}\left(T_{j} V_{j}\right)_{a b}\left(T_{k} V_{k}\right)_{b b^{\prime \prime}}\left(T_{l} V_{l}\right)_{b^{\prime \prime} a} /\left(E_{a}-E_{b}\right)^{2}\left(E_{a}-E_{b^{\prime \prime}}\right) \\
& -\sum_{b b^{\prime}}\left(T_{i} V_{i}\right)_{a b^{\prime}}\left(T_{j} V_{j}\right)_{b^{\prime} b}\left(T_{k} V_{k}\right)_{b a}\left(T_{l} V_{l}\right)_{a a} /\left(E_{a}-E_{b^{\prime}}\right)\left(E_{a}-E_{b}\right)^{2} \\
& +\sum_{b b^{\prime} b^{\prime \prime}}\left(T_{i} V_{i}\right)_{a b^{\prime}}\left(T_{j} V_{j}\right)_{b^{\prime} b}\left(T_{k} V_{k}\right)_{b b^{\prime \prime}}\left(T_{l} V_{l}\right)_{b^{\prime \prime} a} /\left(E_{a}-E_{b}\right)\left(E_{a}-E_{b^{\prime}}\right)\left(E_{a}-E_{b^{\prime \prime}}\right) \\
& \left.+\sum_{b}\left(T_{i} V_{i}\right)_{a a}\left(T_{j} V_{j}\right)_{a b}\left(T_{k} V_{k}\right)_{b a}\left(T_{l} V_{l}\right)_{a a} /\left(E_{a}-E_{b}\right)^{3}\right\} \cdot\left(R_{i} R_{j} R_{k} R_{l}\right)_{K K^{\prime}} \\
& \equiv \frac{1}{\left(\frac{9}{4} F^{0}\right)^{3}} C_{i j k l}\left\{h_{i} h_{j} h_{k} h_{l} /\left(\Delta \Delta^{\prime} \Delta^{\prime \prime}\right)-h_{i}\left(h_{j} h_{k} h_{l} / \Delta^{2} \Delta^{\prime}\right)-\left(h_{i} h_{j} h_{k} / \Delta \Delta^{\prime 2}\right) h_{l}\right. \\
& \left.-\frac{1}{2}\left(h_{i} h_{j} / \Delta\right)\left(h_{k} h_{l} / \Delta^{2}\right)-\frac{1}{2}\left(h_{i} h_{j} / \Delta^{2}\right)\left(h_{k} h_{l} / \Delta\right)+h_{i}\left(h_{j} h_{k} / \Delta^{3}\right) h_{l}\right\} \cdot\left(r_{i} r_{j} r_{k} r_{l}\right)_{K K^{\prime}} \cdot
\end{aligned}
$$

19 Die Indizes an den Operatorensymbolen $H, T V$ und $R$ kennzeichnen in unserem Fall die Teiloperatoren von (36). 
$\Delta$ ist die Differenz der mit $\frac{9}{4} F^{0}$ reduzierten Energien, $C_{i}, C_{i j}, C_{i j k}, C_{i j k l}$ fassen die Koeffizienten aus der jeweiligen Operatorkombination zusammen, $h_{i}$ ist ein Matrixelement eines von konstanten Faktoren getrennten Operators, also etwa von $\cos 3 \alpha, p_{\alpha}, p_{q}$, $p_{a} q$. Wenn $h$ nicht in den Summen $h h / \Delta$ etc. steht, ist es ein Diagonalelement. $r_{i}$ ist ein Matrixelement eines reinen Rotationsoperators, also etwa $P_{g}, P_{g}{ }^{2}$, $P_{g} P_{g^{\prime}}$.

Wegen (49) setzt sich der Gesamtbeitrag der van Vleck-Transformation zusammen aus der Summe über alle Operatorenkombinationen. Ist $n$ die Zahl der Störoperatoren, $\delta$ die Ordnung der jeweiligen van Vleck-Transformationen, so besteht diese Summe aus $n^{\delta}$ Teilsummen (51) - (54). Der Gesamtbeitrag bis vierter Ordnung enthält also $n+n^{2}+n^{3}+n^{4}$ Teilsummen.

Faßt man jeweils die Teilsummen mit gleichem $\left(r_{i}\right)_{K K^{\prime}},\left(r_{i} r_{j}\right)_{K K^{\prime}}, \quad\left(r_{i} r_{j} r_{k}\right)_{K K^{\prime}}$ und $\left(r_{i} r_{j} r_{k} r_{l}\right)_{K K^{\prime}}$

zusammen und ersetzt formal diese Matrixelemente von Rotationsoperatoren durch die Rotationsoperatoren selbst, so erhält man einen effektiven Rotationsoperator $H_{\text {eff }}^{\prime}$, der den Beitrag der van VleckTransformation zur $v_{a} v_{q}$-Submatrix beschreibt.

$$
\begin{gathered}
H_{\text {eff }}^{\prime}=\sum_{\alpha \beta \gamma} K_{v \alpha \sigma v q}^{(\alpha \beta \gamma)}(s, \omega)\left[P_{x}^{\alpha}, P_{y}^{\beta}, P_{z}^{\gamma}\right] \\
K_{v \alpha \sigma v q}^{(\alpha \beta \gamma)}(s, \omega)=\sum_{n=1}^{4} K_{v \alpha \sigma v q}^{(n)(\alpha \beta \gamma)}(s, \omega),
\end{gathered}
$$

$\left[P_{x}^{\alpha}, P_{y^{\beta}}{ }^{\beta}, P_{z^{\prime}}\right]$ steht summarisch für alle möglichen Anordnungen von $\alpha$ Operatoren $P_{x}, \beta$ Operatoren $P_{y}$ und $\gamma$ Operatoren $P_{z}$. Jede dieser Operatorenanordnungen besitzt einen spezifischen Koeffizienten $K_{v \alpha \sigma v_{q}}^{(\alpha \beta \gamma)}$. Die Kennzeichnung $(\alpha \beta \gamma)$ ist hier in gleicher Weise summarisch. $\sum_{\alpha \beta \gamma}$ bezeichnet eine Summation über alle möglichen Operatorenanordnungen beginnend mit $\alpha+\beta+\gamma=1$.

$$
\begin{aligned}
& K_{v \alpha \sigma v q}^{(1)(\alpha \beta \gamma)}(s, \omega)=\sum_{i}^{(\alpha \beta \gamma)} C_{i} h_{i}, \\
& K_{v \alpha \sigma q v}^{(3)(\alpha \beta \gamma)}(s, \omega)=\frac{1}{\left(\frac{9}{4} F^{0}\right)^{2}} \sum_{i j k}^{(\alpha \beta \gamma)} C_{i j k}\left(h_{i} h_{j} h_{k} / \Delta \Delta^{\prime}-\frac{1}{2} h_{i}\left(h_{j} h_{k} / \Delta^{2}\right)-\frac{1}{2}\left(h_{i} h_{j} / \Delta^{2}\right) h_{k}\right), \\
& K_{v \alpha \sigma v q}^{(4)(\alpha \beta \gamma)}(s, \omega)=\frac{1}{\frac{9}{4} F^{0}} \sum_{i j}^{(\alpha \beta \gamma \gamma)} C_{i j}\left(h_{i} h_{j} / \Delta\right), \\
&\left.-\left(h_{i} h_{j} h_{k} / \Delta \Delta^{\prime 2}\right) h_{l}-\frac{1}{2}\left(h_{i} h_{j} / \Delta\right)\left(h_{k} h_{l} / \Delta^{2}\right)-\frac{1}{2}\left(h_{i} h_{j} / \Delta^{2}\right)\left(h_{k} h_{l} / \Delta\right)+h_{i}\left(h_{j} h_{k} / \Delta^{3}\right) h_{l}\right) .
\end{aligned}
$$

$\stackrel{(\alpha \beta \gamma)}{\Sigma}$

$\stackrel{(\alpha \beta \gamma)}{\Sigma}$ zeigt an, daß nur solche Beiträge aufsummiert werden, deren Rotationsanteil sich durch die Operatoren $P_{x}^{\alpha} P_{y}^{\beta} P_{z}{ }^{\gamma}$ repräsentieren läßt. $\sigma$ bezeichnet die Torsionsspezies.

Da die $C_{i}, C_{i j}, C_{i j k}, C_{i j k l}$ Potenzen von $V_{\mathrm{c}}^{\prime} / F^{0}$ enthalten können, ist jeder Koeffizient $K_{v \alpha \sigma v_{q}}^{\left(\alpha \beta \gamma_{q}\right)}(s, \omega)$ ein Polynom in $V_{\mathrm{c}}{ }^{\prime} / F^{0}$.

Die $K_{v \alpha \sigma v)}^{\left(\alpha \beta_{\alpha} \gamma\right.}(s, \omega)$ sind außerdem Funktionen vom reduzierten Hinderungspotential $s$, der Schwingungsfrequenz $\omega$, von der Struktur des Moleküls, von den Massen der Atome und der Schwingungsform.

Es verbleibt nun noch die Aufgabe, die Matrix des effektiven Rotationsoperators

$$
H_{\text {eff }}=C^{0} P_{x}^{2}+B^{0} P_{y}^{2}+A^{0} P_{z}^{2}+H_{\text {eff }}^{\prime}
$$

zu diagonalisieren. Diese Aufgabe läßt sich selbst für hohe $J$ numerisch gut bewältigen.

Da die Koeffizienten des effektiven Operators (58) Zahlen sind, hat er die gleiche Form wie der effektive Operator, der aus dem einfachen Modell ohne Schwingung resultiert. Beobachtet man nur das Ro- tationsspektrum in einem Zustand $v_{a} v_{q}$, so ist die Wiedergabe des Spektrums kein Maß für die Güte des Modells. Die Güte des Modells erweist sich erst in der Wiedergabe der Rotationsspektren mehrerer Zustände.

Zur numerischen Behandlung von Operatoren der Art von (36) wurde ein Rechenprogramm geschrieben, das neben Eigenwerten und Fourier-Koeffizienten der Eigenfunktionen des Mathieu-Operators (39) Matrixelemente $p_{a}, p_{\alpha}^{2}, \cos 3 \alpha, \sin 3 \alpha, \cos 6 \alpha$, $\sin 6 \alpha$ und Störsummen der Typen

$h_{i} ; \quad h_{i} h_{j} / \Delta^{n} ; \quad h_{i} h_{j} h_{k} / \Delta^{n} \Delta^{\prime m} ; \quad h_{i} h_{j} h_{k} h_{l} / \Delta \Delta^{\prime} \Delta^{\prime \prime}$ berechnet. Die $h_{i}$ können dabei aus einem Produkt eines Torsions- und eines Schwingungsoperators erzeugt werden. Für Torsionsoperatoren stehen die Operatoren $1, p_{\alpha}, p_{a}{ }^{2}, \cos 3 \alpha, \sin 3 \alpha, \cos 6 \alpha, \sin 6 \alpha$ für Schwingungsoperatoren $1, p_{q}, p_{q}{ }^{2}, q, q^{2}, p q, q p$ zur Wahl. Hilfsprogramme besorgen die Erstellung von Eingabeanweisungen für alle Kombinationen $i$, $i j$, ijk, ijkl einer Operatorenwahl und dienen zur Aufsummierung der einzelnen Störsummen. 
Das Programm verwendet die Symmetrie der Störsummen

$$
\begin{aligned}
h_{i} h_{j} / \Delta^{n} & =h_{j} h_{i} / \Delta^{n}, \\
h_{i} h_{j} h_{k} / \Delta^{n} \Delta^{\prime m} & =h_{k} h_{j} h_{i} / \Delta^{m} \Delta^{\prime n}, \\
h_{i} h_{j} h_{k} h_{l} / \Delta \Delta^{\prime} \Delta^{\prime \prime} & =h_{l} h_{k} h_{j} h_{i} / \Delta \Delta^{\prime} \Delta^{\prime \prime} .
\end{aligned}
$$

Das Programm ist in Fortran IV geschrieben und rechnet mit doppelter Wortlänge.

In einer folgenden Arbeit wird die Anwendung der gegebenen theoretischen Formulierungen auf das Rotationsspektrum von Methylthiocyanat gegeben.

Ich danke Herrn Dr. D. Sutrer, Dr. H. D. Rudolph, Freiburg, Frau Dr. A. Mirri und Prof. Dr. P. Favero, Bologna, für viele Diskussionen. Die Arbeit wurde von der Deutschen Forschungsgemeinschaft und dem Fonds der Chemie unterstützt. Herrn Prof. Dr. P. Favero und dem Centro Nazionale delle Ricerche, Rom, danke ich für einen mehrmonatigen Forschungsaufenthalt in Bologna. 